\title{
CMS Muon Reconstruction and Identification Performance of Run2 Data
}

\author{
Sihyun Jeon*t \\ on behalf of CMS collaboration \\ E-mail: shjeon@cern.ch
}

\begin{abstract}
The Compact Muon Solenoid (CMS) detector is one of the 4 detectors at the CERN LHC to study the Standard Model (SM) and even searching for signatures of new physics produced in highenergy collisions in the LHC. As many of the signatures in both SM and new physics include muons, it is crucial to possess high performance of muon identification and reconstruction. The performance of CMS muon identification and reconstruction is studied based on the run 2 data collected with the CMS detector at $\sqrt{s}=13 \mathrm{TeV}$. The identification and reconstruction efficiencies are measured using tag and probe method. As High-Level Trigger (HLT) has made improvements in its algorithms during the run 2, we also highlight its upgraded feature by comparing with the previous algorithm.
\end{abstract}

The 39th International Conference on High Energy Physics (ICHEP2018)

4-11 July, 2018

Seoul, Korea

\footnotetext{
* Speaker.

${ }^{\dagger}$ Seoul National University
} 


\section{CMS Muon System}

The CMS muon system consists of 3 different gaseous detectors, drift tubes, cathode strip chambers, and resistive plate chambers.

\section{Muon Identification and Isolation}

Identification and isolation criteria are applied to reconstructed muons to remove fake (nonprompt) muons. These efficiencies are measured using run 2 data as shown in Fig. 1.
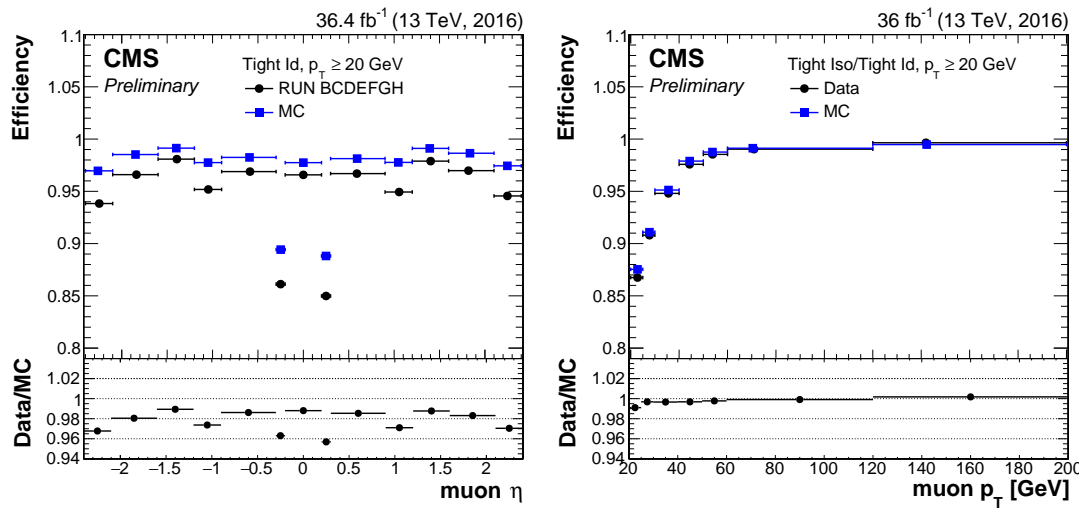

Figure 1: Muon identification (left) and isolation efficiencies (right)

\section{Improvements in Muon HLT}

In 2018, IterL3 algorithm was updated to mainly improve the efficiency in HLT level. Fig. 2 shows the efficiencies of HLT comparing before and after updating IterL3 algorithm.
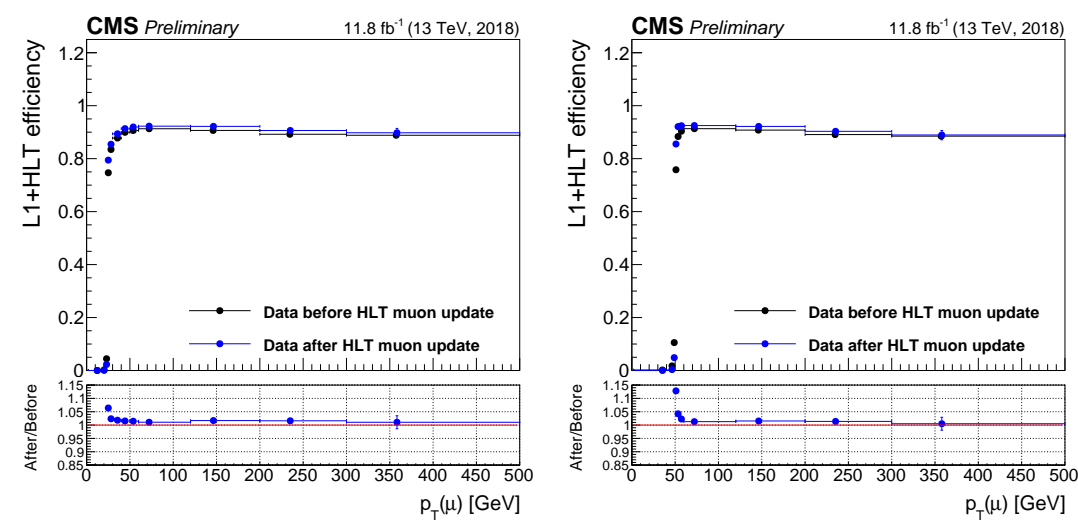

Figure 2: Trigger efficiency of isolated (left) and nonisolated muon triggers (right)

\section{References}

[1] The CMS Collaboration, Performance of the CMS muon detector and muon reconstruction with proton-proton collisions at $\sqrt{s}=13 \mathrm{TeV}$, Journal of Instrumentation, 13 (2018) P10002. 